Send your letters to the Editor,

British Dental Journal,

64 Wimpole Street

London

W1G 8YS

E-mail bdj@bda.org

Priority will be given to letters less than 500 words long.

Authors must sign the letter, which

may be edited for reasons of space.

\section{CONFIRM THE EFFICACY}

Sir, we would like to comment on the recently published National Institute for Clinical Excellence (NICE) guidelines on infective endocarditis (IE). ${ }^{1}$

With regard to 'at risk' cardiac conditions of IE, three Expert Committees (NICE, AHA and BSAC) agree in defining a single category of 'high-risk' patients requiring prophylaxis, but the cardiac conditions included by each committee differ. This lack of consensus could cause conflicting situations for clinicians at the time of identifying 'highrisk' patients, with possible medico-legal repercussions. Conversely, if a clinician considers the 'high-risk' cardiac conditions all in one category this does not reduce the 'at risk' conditions compared with previous IE protocols.

In relation to the effect of antibiotic prophylaxis on post-dental manipulation bacteraemia, NICE concluded: Antibiotic prophylaxis does not eliminate bacteraemia following dental procedures...' This conclusion was reached after analysis of studies that presented methodological differences with respect to the type of antibiotic used, and the time and route of administration, and it is inappropriate to compare the results between the series. In the majority of the studies published on antibiotic prophylaxis and post-dental extraction bacteraemia, the authors confirm the efficacy of the penicillins, as bacteraemia did not develop in a significant number of patients. ${ }^{2,3}$ However, there are fewer studies on the effect of other recommended antibiotics (clindamycin, azithromycin and cephalosporins) and their results do not establish whether these antibiotics are effective. ${ }^{2}$

In a second conclusion NICE states: 'It is not possible to determine the effect of antibiotic prophylaxis on the duration of bacteraemia'. Few studies have been published on the effect of antibiotic prophylaxis on the duration of post-dental extraction bacteraemia. $^{2}$ We have demonstrated that the administration of oral amoxicillin significantly reduces the duration of post-extraction bacteraemia. ${ }^{3}$

With regard to the effect of chlorhexidine prophylaxis on post-dental manipulation bacteraemia, NICE concluded: 'Chlorhexidine used as an oral rinse does not significantly reduce the level of bacteraemia following dental procedures'. This conclusion has been reached after analysis of studies that presented methodological differences with respect to the dental procedure performed, the concentration of chlorhexidine used, and the technique for applying the antiseptic, and it is inappropriate to compare the results between these series. There are very few studies in the literature on the efficacy of mouth rinsing with $0.2 \%$ chlorhexidine (recently recommended by the BSAC in 2006) for the prevention of post-dental extraction bacteraemia. ${ }^{4}$ We have demonstrated that initial rinsing with $0.2 \%$ chlorhexidine significantly reduced the duration of post-dental extraction bacteraemia. ${ }^{5,6}$

The conclusions reached by NICE on the lack of efficacy of antimicrobial prophylaxis for the prevention of postdental manipulation bacteraemia are based on scarce published evidence. Further research should therefore be performed on the recommended antibiotics regimens, analysing the influence of the choice of antibiotic, and the time and route of administration and also on new antibiotic protocols. At the present time, the controversies about the risk of developing IE of oral origin, the clinical repercussions of bacteraemia of oral origin, the efficacy of antibiotic prophylaxis, and the riskbenefit and cost-benefit relationships, could justify the current reappraisal on the need for IE antibiotic prophylaxis. In consequence, further research should be encouraged to confirm the efficacy of the recommended chlorhexidine regimens and to investigate new antiseptic protocols.

I. Tomás, J. Limeres, P. Diz

Spain

1. NICE guidelines on prophylaxis against infective endocarditis. http://www.nice.org.uk/nicemedia/ pdf/PIEGuidelines.pdf

2. Tomás Carmona I, Diz Dios P, Scully C. Efficacy of antibiotic prophylactic regimens for the prevention of bacterial endocarditis of oral origin. J Dent Res 2007; 86: 1142-1159.

3. Diz Dios P, Tomás Carmona I, Limeres Posse Jet al. Comparative efficacies of amoxicillin, clindamycin and moxifloxacin in prevention of bacteremia following dental extractions. Antimicrob Agents Chemother 2006; 50: 2996-3002.

4. Lockhart P B. An analysis of bacteremias during dental extractions. A double-blind, placebo-controlled study of chlorhexidine. Arch Intern Med 1996: 156: 513-520.

5. Tomás I, Álvarez M, Limeres J et al. Effect of chlorhexidine mouthwash on the risk of post-extraction bacteremia. Infection Control Hosp Epidemiol 2007: 28: 577-582.

6. Diz P, Tomás Carmona I, Barbosa M et al. A chlorhexidine mouthwash reduces the risk of bacteraemia following dental extractions performed under either general or local anaesthesia. Clin Res Cardiol 2007; 96: 443-444.

DOI: 10.1038/sj.bdj.2008.568

\section{OVERSEAS REGISTRATION}

Sir, I am writing in regard to the International English Language Testing System (IELTS) score required before sitting the GDC's Overseas Registration Examination (ORE).

I have taken the IELTS three times over the past six months, however, I was unlucky in the writing category in the first and second exams, 
getting score 6, although I had 7s and $8 \mathrm{~s}$ in the others. The third time I managed to get 7 in writing but it was the reading which, purely due to time, was 6. Listening and speaking were 7 and 8 respectively. I am not asking the GDC to lower the score they want; instead, I was hoping that they might consider looking at these three exams rather than just one exam.

I am surprised and puzzled by the fact that EU dentists are not required to take the IELTS at all because they come from the EU. However, a dentist like me, from Libya, who has been in the UK for about ten years (doing Masters and a $\mathrm{PhD}$ ) is required to sit such an exam. In fact, if I became a British citizen, I would not be required to do the exam when my English level would still be the same.

I have always thought of the GDC as being there to protect the patients and maintain high standards of dental care, but it seems that political decisions have come in between, as someone from an EU country, whose English is not that good, can come to the UK and practice with no need to sit IELTS. This does not, at all, show any care for the poor patient who will be struggling to explain their problem, and therefore, you are watching this situation taking place and doing nothing, which can be considered neglect.

T. Elgalaid

Newcastle

DOI: 10.1038/sj.bdj.2008.569

\section{SOCIO-DENTAL MEASURE}

Sir, I write in reference to the paper Oral health-related quality of life and the IOTN index (BDJ 2008; 204: E12).

As a member of the original Bedfordshire PDS pilot from which the data for the above paper were derived, I read the paper with great interest and would like to comment on a number of points. I noted with a degree of dismay that there were a number of patients offered treatment despite little or no dental health need and also when the patient did not report any dental health impact. This does not reflect in the data collected by the PDS which explicitly required patients who embarked upon orthodontic treatment, to have an IOTN DHC 3 AC
6 or above. Only in exceptional circumstances, when permission from the PCT had been granted, were patients below these criteria treated.

In the paper it is assumed patients placed on review were being accepted for treatment even if the IOTN was below the PDS criteria. I would argue that this may not be an appropriate measure. Patients with a low IOTN may warrant being placed on review ... such as the management of dubious first molars, monitoring adverse skeletal growth in the growing class III malocclusion or monitoring dental development in patients in the late mixed dentition where there is the potential for crowding ... but these cases may end up being treated. These review patients would have a low IOTN, may not have a perceived need for braces, but would be placed in the treatment offered/little borderline need category. This is important because some of your conclusions reflect upon the inadequacies of the current system offering treatment to patients with little/borderline dental health need and in which the patient has no perceived need for a brace.

Asking for the child's perceived need for orthodontic treatment at this stage, also, is of concern. In life one's perception of need can be influenced by a host of appropriate or inappropriate life experiences. In the current study the patients will have already been seen by a dentist and referred, presumably creating or reinforcing the notion that there were problems with their teeth and raising the expectation that they were in need of a brace. Indeed some at this point may have been misled into the view that they had a dental impact and needed a brace, even in the absence of a significant dental problem. In such a case the denial of orthodontic treatment may have been appropriate.

The initial assessment, involving the IOTN score, is only the first step in progressing to orthodontic treatment. Other factors such as caries, poor $\mathrm{OH}$ etc may mean it is inappropriate to offer treatment to a patient. Once patients have been identified as appropriate for NHS treatment within the PDS criteria, it would be standard practice for a more exact treatment plan to be formulated and discussed with the patient and parent. At this point the option of doing nothing is discussed and patients are free to express their desire or otherwise for treatment. One would hope that at this point those patients who had a need, and once informed about that need, did not want a brace, would not get one. Or conversely those who thought they did not need a brace might, once faced with information regarding their malocclusion, opt for treatment. It is at this point that I would like to know which patients I am failing and if an oral health related quality of life measure would be of use. I don't think the study has addressed this, nor do I think it has made a sound case that it is essential to incorporate a socio-dental measure into the evaluation of need of orthodontic treatment.

\section{J. S. K. Evans}

On behalf of the Bedfordshire Orthodontic PDS Pilot DOI: 10.1038/sj.bdj.2008.570

\section{UNUSUAL FUSION}

Sir, we would like to share with your readers an unusual case of a patient with a mesiodens fused to the adjacent central incisor in the mandibular midline.

A 27-year-old male visited our department for a routine dental check up. Clinical examination revealed an extra tooth in the mandibular midline (Fig. 1). This tooth was found lingual to and overlapping the mesial aspect of the right central incisor. We placed a matrix band and dental floss between the teeth to determine if there was fusion of the crown portion with the adjacent tooth, but the crowns were separated. Based on this finding we provisionally thought of an erupted mesiodens. However, an IOPA radiograph (Fig. 2) revealed a striking finding. There was overlap of the coronal portion of the extra tooth with the mesial aspect of the mandibular right central incisor and the radicular portions of both teeth were fused to each other. The radiograph clearly revealed two separate pulp canals joined at the apex of the tooth giving the appearance of fusion. Fusion is defined as a single enlarged tooth or joined teeth in which the tooth count reveals a missing tooth when the anomalous tooth 


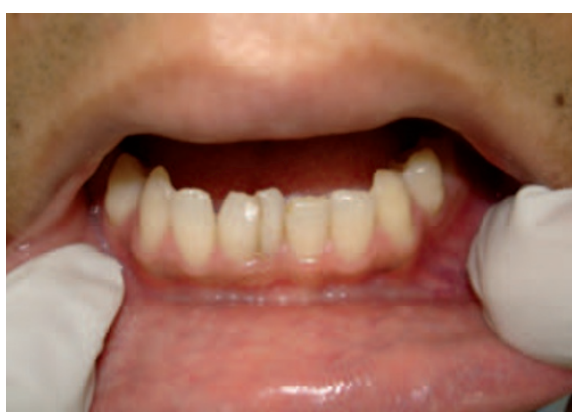

Fig. 1 Extra tooth in the mandibular midline

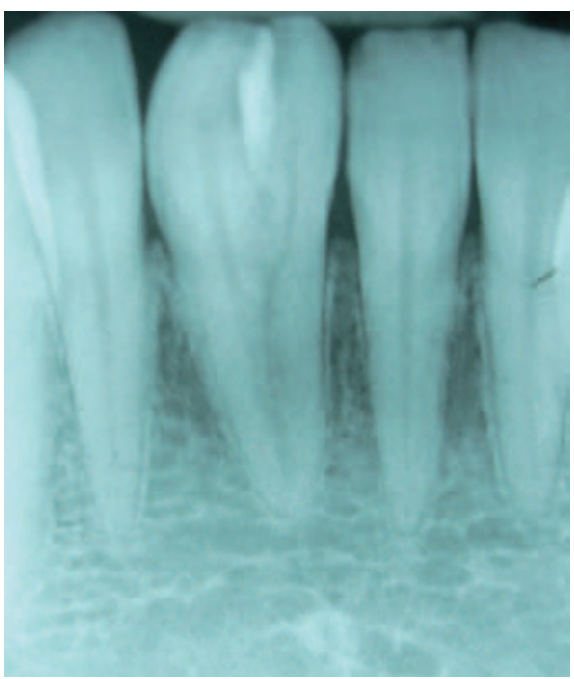

Fig. 2 Radiograph showing the overlap of the coronal portion of the extra tooth

is counted as one. ${ }^{1,2}$ Gemination was excluded from the diagnosis because according to most authors gemination usually presents with a single root canal. ${ }^{1}$ Separate root canals are usually found in fusion, however, this does not hold true for all cases. The present case is quite unusual as very few cases have been reported in the literature. A large percentage of anterior supernumerary teeth remain unerupted (75\%) while $25 \%$ are partially erupted. ${ }^{3}$ In our case the mesiodens was completely erupted. The overall prevalence of mesiodens is $0.15 \%$ to $1.9 \% .{ }^{4}$ We suggest that a new terminology should be given to such cases of fusion of a supernumerary tooth with an adjacent tooth to avoid confusion between fusion and gemination.

\section{A. A. Sholapurkar \\ K. M. Pai \\ Manipal}

1. Neville B N, Damm D D, Allen C M, Bouquot J E. Oral and maxillofacial pathology, 2nd ed. pp 7476. Philadelphia: WB Saunders, 2002

2. Uÿs H, Morris D. 'Double' teeth - a diagnostic conundrum. Dent Update 2005; 32: 237-239.

3. Sharma S A. Mandibular midline supernumerary tooth: a case report. J Indian Soc Pedod Prev Dent 2001; 19: 143-144.

4. Russell K A, Folwarczna M A. Mesiodens - diagnosis and management of a common supernumerary tooth. J Can Dent Assoc 2003; 69: 362-366.

DOI: 10.1038/sj.bdj.2008.571

\section{GAG TECHNIQUE}

Sir, after reading the letter on the management of TMJ dislocation by Nusrath et al. (BDJ 2008; 204: 170-171) I would like to share with readers a technique that I have found useful.

This was taught to me some 20 years ago by my teacher, Associate Professor Dr Mohd Nor Awang who described and reported three cases of reducing dislocated TMJs using the gag reflex. ${ }^{1}$ This technique simply requires you to touch the end of a dental mirror onto the patient's uvula to induce gagging. In reaction to the gagging the patient will subconsciously move the mandible and before realising it, it will be back in position.

I have tried this technique on at least ten occasions and found that it works well if a dislocated mandible is seen and attended to within the first 30 minutes. Otherwise, muscle spasm will set in, and even though the patient still gags, this action will not reduce the mandible. Therefore, before even attempting manual manipulation, which is what we are usually taught (but not shown) in dental school, try inducing the gag reflex instead? I have also suggested to patients with recurrent dislocations to try tickling their own throat with their finger to create the same effect. Lastly, patients may gag but will not vomit and nor will the jaw snap onto the mouth mirror!

W. C. Ngeow

Kuala Lumpur

1. Awang M N. A new approach to the reduction of acute dislocation of the temporomandibular joint: a report of three cases. Br J Oral Maxillofac Surg 1987; 25: 244-249.

DOI: $10.1038 /$ sj.bdj.2008.572

\section{MEDICAL AND DENTAL GOLD}

Sir, in response to T. Sebastian's letter (BDJ 2008; 204: 545), as a Muslim dentist, I would like to explain that in Islam it is allowed for males to use gold and silver for medical reasons only. The use of gold and silver is prohibited for males if used as jewels for example as finger rings and so forth.

0. Menla

By email

DOI: 10.1038/sj.bdj.2008.573

\section{UNNECESSARY WASTE}

Sir, in January I sent an email to $\mathrm{Mr}$ Rudkin, Registrar of the General Dental Council. In it I raised the issue of unnecessary waste by the GDC when dealing with the registration of dentists and the future registration of DCPs.

In November 2007 I received notification of the need to renew my registration by 31 December. I received a reminder in December. My dental nurse who was already registered received further registration information. I suspect this was not a unique occurrence.

I expressed my opinion that this duplication of correspondence was totally unjustified, was a waste of our, the registrants', money, and was a scandalous environmental waste.

It is my view that it is the individual's responsibility to ensure that his/her registration is renewed in time. Reminders should not be required. I have managed to renew mine annually for the past 35 years.

After some prompting and resubmission of my email I received an acknowledgement of its receipt but as yet I have received no reply.

To me this is a further example of the increasingly arrogant attitude of the GDC towards its registrants. I suggest that it is perhaps time for the profession to carry out a critical analysis of its regulatory body.

J. Aitken Kenilworth DOI: $10.1038 /$ sj.bdj.2008.574 